\title{
PRODUCTIVIDAD, RESULTADO ECONÓMICO Y RIESGO DE SISTEMAS LECHEROS EN EL CENTRO-NORTE DE ARGENTINA
}

\section{PRODUCTIVITY, ECONOMIC RESULTS AND RISK OF DAIRY SYSTEMS IN NORTHERN-CENTRAL ARGENTINA}

\author{
Javier Baudracco ${ }^{1 *}$, José Maiztegui ${ }^{1}$, José Jáuregui ${ }^{1}$, Belen Lazzarini ${ }^{1}$, Alejandro Rosset ${ }^{1}$, y Roberto \\ Gagliardi $^{2}$ \\ ${ }^{1}$ Facultad de Ciencias Agrarias, Universidad Nacional del Litoral, Kreder 2805, Esperanza, CP: \\ S3080HOF, Argentina. \\ ${ }^{2}$ Cluster Lechero Regional, Guemes 127, San Guillermo, Santa Fe, Argentina. \\ * Autor para correspondencia: jbaudracco@yahoo.com
}

\section{RESUMEN}

Las variaciones en el precio de la leche y de los alimentos concentrados y el efecto del clima sobre la producción de cultivos afectan la productividad rentabilidad y riesgo de los sistemas lecheros. El objetivo del estudio fue investigar la productividad, el resultado económico y el riesgo de diversos sistemas de producción de leche en la región Centro-Norte de Argentina. Se investigaron sistemas lecheros alternativos al sistema representativo de la región, explorando el efecto de incrementos en carga animal, incrementos en suplementación con alimentos concentrados y el efecto de utilizar diferentes sistemas de alimentación sobre la productividad y la rentabilidad. El enfoque elegido para el análisis fue la simulación con el uso de dos modelos matemáticos: uno para predicción del rendimiento de cultivos y otro para evaluación del sistema lechero completo. Se realizaron simulaciones determinísticas y estocásticas, i.e., con variación aleatoria del precio de la leche, de los concentrados y el rendimiento de los cultivos. Los resultados muestran que el sistema actual de la región no es rentable. Los sistemas alternativos con mayor carga animal y mayor suplementación resultaron en mayor producción de leche por hectárea y mayor resultado económico que el sistema regional actual. Sin embargo, la intensificación a partir del incremento de la carga animal y de la suplementación aumentó la exposición al riesgo. El efecto de la variación de precio de leche y concentrado fue mayor que el efecto del clima sobre el resultado económico.

Palabras clave: estocástico, carga animal, modelo, precios, clima, leche.

\begin{abstract}
Variations in milk and concentrate feed prices and the effect of climate on crop production affect productivity and profitability of dairy systems. The objective of this study was to evaluate productivity and economic results, and assess the risk of different dairy systems in the Central-Northern region of Argentina. Alternative dairy systems (other than the traditional system of this region) were studied, evaluating the effects of increases in stocking rate and use of supplementary feed, as well as the effect of using different feeding systems on productivity and profitability. Two mathematical models were used in this analysis: one model to predict crop and pasture production, and the other to evaluate the performance of the whole dairy system. Deterministic and stochastic simulations, i.e., with random variation in milk and concentrate feed prices, and crop yield, were performed. Results showed that the current production system of this region is not profitable. The alternative systems
\end{abstract}


with higher stocking rate and supplementation resulted in higher milk production per hectare and improved economic results. However, intensification of the system, through increases in stocking rate and supplementation resulted in an increased risk. Milk and concentrate feed prices had more impact on the economic results than climate.

Key words: stochastic, stocking rate, model, prices, climate, milk.

\section{INTRODUCCIÓN}

La lechería en Argentina ocupa un lugar importante en la economía nacional, con una producción de leche de aproximadamente 11.215 millones de litros en el año 2015 (Ministerio de Agroindustria de la Nación, 2016).

Uno de los principales desafíos de los sistemas lecheros es lograr rentabilidad positiva ante variaciones en el precio del producto (leche), de los insumos (principalmente alimentos concentrados) y ante variaciones del clima. Existe una creciente incertidumbre entre los productores lecheros de Argentina, respecto a la definición del sistema productivo que les permita producir en el futuro, en forma rentable y sustentable.

Este desafío es aún más crítico en la región Centro-Norte de Argentina, la cual cuenta con restricciones edafo-climáticas para la producción de leche (Jáuregui et al., 2015). En esta cuenca hay 987 lecherías, que producen en promedio 1.215 litros por día, lo cual es inferior al promedio de Argentina, que produce 2.870 litros de leche por lechería por día (Ministerio de Agroindustria de la Nación, 2016).

Para investigar diferentes sistemas de producción son muy útiles los ensayos a campo (Macdonald et al., 2011), aunque son costosos y por lo tanto se limita la cantidad de sistemas a explorar. Otra alternativa es utilizar modelos de simulación, los cuales son herramientas computacionales que simulan matemáticamente el comportamiento productivo y/o económico de un sistema ante manejos alternativos y escenarios probables (Beukes et al., 2008).

El objetivo del presente estudio fue investigar, mediante estudios de simulación, la productividad, resultado económico y riesgo de diversos sistemas productivos, generados a partir de combinaciones de oferta de alimentos, demanda de alimentos, sistemas de alimentación y biotipo animal para la región Centro-Norte de Argentina.

\section{MATERIALES Y MÉTODOS}

\section{Características de la región bajo estudio}

La Región tiene un clima subtropical-templado, con características edáficas e hídricas, que limitan la producción de forrajes y consecuentemente la producción de leche. Los suelos tienen principalmente limitantes de infiltración y de nutrientes. Los veranos tienen temperaturas máximas promedio de $32^{\circ} \mathrm{C}$ y un balance hídrico negativo debido a la alta demanda atmosférica. Las precipitaciones anuales de la región son en promedio de $998 \mathrm{~mm}$, con alta variación interanual y concentradas en primavera-verano.

\section{Selección de sistemas productivos a evaluar Metodología de trabajo}

El estudio fue diseñado para investigar sistemas lecheros alternativos al sistema representativo de la región bajo estudio. Para definir y evaluar alternativas mejoradoras del sistema lechero actual de la región, se conformó un equipo técnico integrado por 4 investigadores, 4 productores y 4 asesores. La investigación se orientó hacia la problemática del productor, de manera de alcanzar, al final del proceso, conclusiones de impacto para los productores lecheros y sus empresas.

Inicialmente, el equipo técnico definió las principales preguntas de investigación sobre sistemas de producción de leche para la región bajo estudio, la cual se ubica en el norte de la Provincia de Santa Fe y Sur de la Provincia de Santiago del Estero. En base a estas preguntas se formularon 10 sistemas productivos. Luego de definir los sistemas, se efectuaron estudios de simulación utilizando modelos matemáticos diseñados para evaluar la producción de pasturas y cultivos, la producción de leche, el resultado económico y el riesgo de los 10 sistemas lecheros definidos.

\section{Generación de sistemas productivos}

Se definió un sistema denominado BASE, el cual representa el sistema de producción de leche actual, promedio de la región bajo análisis. Luego se generaron 9 sistemas a partir de diferentes combinaciones de las siguientes variables: tipo de suelo, precipitación, carga animal, sistema de alimentación, biotipo animal y cantidad de alimento concentrado usado para alimentar las vacas (Tabla 1$)$.

Precipitación. La región bajo estudio se dividió en dos, de acuerdo al régimen pluviométrico, una zona denominada de precipitación BAJA, 
Tabla 1. Precipitación, tipo de suelo, producción de cultivos, sistema de alimentación, alimento ofrecido, biotipo animal y carga animal de los sistemas lecheros simulados para la región Centro-Norte de Argentina.

Table 1. Precipitation, soil type, crop production, feeding system, feeds offered, animal type and stocking of modeled dairy systems for the Central-North region of Argentina.

\begin{tabular}{|c|c|c|c|c|c|c|c|c|c|c|}
\hline Sistema & 1 (Base) & 2 & 3 & 4 & 5 & 6 & 7 & 8 & 9 & 10 \\
\hline Precipitación $^{1}$ & Baja & Baja & Baja & Baja & Baja & Baja & Baja & Alta & Alta & Alta \\
\hline Suelo ${ }^{2}$ & Bueno & Bueno & Bueno & Bueno & Bueno & Bueno & Regular & Regular & Regular & r Bueno \\
\hline $\begin{array}{l}\text { Producción alfalfa } \\
\qquad(\mathrm{kg} \text { MS ha-1 por año) }\end{array}$ & 8.000 & 8.000 & 8.000 & 8.000 & 8.000 & 8.000 & 6.000 & 6.500 & 6.500 & 10.000 \\
\hline $\begin{array}{l}\text { Producción maíz/sorgo } \\
\text { ensilaje }\end{array}$ & & & & & & & & & & \\
\hline (kg MS ha-1 por año) & 8.000 & 8.000 & 8.000 & 8.000 & 8.000 & 8.000 & 6.500 & 7.500 & 7.500 & 11.000 \\
\hline Avena & & & & & & & & & & \\
\hline (kg MS ha-1 por año) & 4.000 & 4.000 & 4.000 & 4.000 & 4.000 & 4.000 & 2.800 & 3.000 & 3.000 & 5.000 \\
\hline Sistema alimentación ${ }^{3}$ & Pasto & Pasto & Pasto & $\begin{array}{l}\text { Pasto y } \\
\text { Autoc. }\end{array}$ & Pasto & Corral & Pasto & Pasto & Pasto & Pasto \\
\hline Tipo vaca ${ }^{4}$ & $\begin{array}{c}\mathrm{H} 580 \\
\mathrm{~kg}\end{array}$ & $\begin{array}{c}\mathrm{H} 580 \\
\mathrm{~kg}\end{array}$ & $\begin{array}{c}\mathrm{H} 580 \\
\mathrm{~kg}\end{array}$ & $\begin{array}{l}\mathrm{H} 580 \\
\mathrm{~kg}\end{array}$ & $\begin{array}{c}\text { Genial }^{5} \\
\mathrm{~kg}\end{array}$ & $\begin{array}{c}\mathrm{H} 700 \\
\mathrm{~kg}\end{array}$ & $\begin{array}{c}\mathrm{H} 580 \\
\mathrm{~kg}\end{array}$ & $\begin{array}{c}\mathrm{H} 580 \\
\mathrm{~kg}\end{array}$ & $\begin{array}{l}\mathrm{H} 580 \\
\mathrm{~kg}\end{array}$ & $\begin{array}{c}\mathrm{H} 580 \\
\mathrm{~kg}\end{array}$ \\
\hline $\begin{array}{l}\text { Carga animal }{ }^{6} \\
\left(\mathrm{VT} \mathrm{ha}^{-1}\right)\end{array}$ & 1,0 & 1,3 & 1,6 & 1,6 & 1,7 & 1,7 & 0,8 & 0,8 & 1,5 & 1,3 \\
\hline Cantidad de vacas & 150 & 195 & 240 & 240 & 255 & 255 & 120 & 120 & 225 & 195 \\
\hline $\begin{array}{l}\text { Concentrado } \\
\left.\text { (kg MS vaca }{ }^{-1} \text { por día }\right)\end{array}$ & 4,0 & 5,5 & 7,0 & 7,0 & 7,0 & 10,0 & 4,0 & 4,0 & 7,0 & 5,0 \\
\hline $\begin{array}{l}\text { Heno comprado } \\
\left.\qquad \text { (kg MS vaca }^{-1} \text { por día }\right)\end{array}$ & - & - & - & - & - & - & - & - & 2,5 & - \\
\hline
\end{tabular}

${ }^{1}$ Precipitación BAJA corresponde a la zona Oeste de la región bajo estudio, con un promedio de 912 mm en los últimos 50 años, en la que se encuentra la mayor cantidad de lecherías. Precipitación ALTA corresponde a la zona Este de la región bajo estudio, con un promedio de $1.085 \mathrm{~mm}$ en los últimos 50 años

${ }^{2}$ Suelo BUENO y suelo REGULAR. Se simularon producciones de forrajes en base a dos tipos de suelos característicos de la región. A los fines de la simulación, la diferencia entre suelos BUENO y REGULAR es su potencial de producción de forrajes, en $\mathrm{kg}$ de materia seca (MS).

${ }^{3}$ Sistema de alimentación. Pasto: vacas en pastoreo; Corral: vacas en confinamiento (corral a cielo abierto) todo el año, sin acceso a pastoreo. Pasto y autoc.: vacas en pastoreo, con consumo de ensilaje en autoconsumo.

${ }^{4}$ Biotipo animal. H580 kg: vaca Holstein de Argentina con $580 \mathrm{~kg}$ de peso promedio; H700 kg: vaca Holstein Americana, de $700 \mathrm{~kg}$ de peso promedio.

${ }^{5}$ Genial: vaca Holstein mejorada en Argentina (Gagliardi, 2012).

${ }^{6}$ Carga animal: cantidad de vacas por hectárea; VT: vacas totales.

${ }^{7}$ Heno comprado: comprado fuera de la superficie del campo (kg de materia seca por vaca día ${ }^{-1}$ )

con un promedio de $912 \mathrm{~mm}$ por año, y otra zona denominada de precipitación ALTA, con un promedio de $1.085 \mathrm{~mm}$ por año, ambos en los últimos 50 años.

Tipo de suelo. En la región existen suelos con distinto potencial productivo de forrajes. A los fines de la simulación, se definieron dos situaciones: Suelo BUENO y suelo REGULAR. La diferencia entre suelo BUENO y REGULAR fue su potencial de producción de forrajes (Tabla 1). La estimación de producción de forraje esperada se hizo a partir de datos de ensayos locales combinada con el aporte de la experiencia de los técnicos de la región.
Sistema de alimentación. Esta variable hace referencia a la forma de suministro de los alimentos y al tipo de alojamiento de los animales. Así, se definieron tres sistemas de alimentación: i) PASTO: vacas en pastoreo, suplementadas con concentrados y ensilaje suministrado con mixer (carro mezclador de alimentos); ii) CORRAL: vacas en confinamiento (corral a cielo abierto) todo el año, sin acceso a pastoreo, con dieta totalmente mezclada (ensilaje, heno y concentrado), y iii) PASTO y AUTOCONSUMO: vacas en pastoreo, con suministro de ensilaje en autoconsumo y concentrado en sala de ordeño. El autoconsumo de ensilaje es una técnica desarrollada para vacas lecheras en la Provincia de Santa Fe, que consiste 
en el acceso directo de las vacas a las bolsas de ensilaje (Baudracco et al., 2016), evitando la extracción, transporte y distribución del ensilaje. Esta forma de suministro reduce la demanda laboral y los costos de distribución de alimentos.

Carga animal. Hace referencia a la cantidad de vacas totales (vacas en ordeño + vacas secas) en la superficie dedicada a las vacas totales (VT). La superficie de VT se define como la superficie destinada a pastura y a producción de reservas voluminosas (no incluye superficie dedicada a producir granos, aunque se usen para el consumo de las vacas). Las cargas animales utilizadas variaron entre 0,8 y $1,7 \mathrm{VT} \mathrm{ha}^{-1}$.

Biotipo animal. Se refiere al biotipo animal dentro de la raza Holstein (Ver Tabla 1). En este estudio se utilizaron tres biotipos diferentes: vacas Holstein con $580 \mathrm{~kg}$ de peso vivo (H580), vacas Holstein de $700 \mathrm{~kg}$ de peso vivo (H700), y vacas Holstein de $540 \mathrm{~kg}$ de PV.

Cantidad de concentrado. Se refiere a la cantidad de concentrados ofrecido por vaca por día. Los niveles de concentrado suministrado variaron entre 4,0 y 10,0 kg por vaca por día (Tabla 1).

La combinación de estas variables dio como resultado la generación de 10 sistemas productivos (Tabla 1), el sistema BASE y 9 sistemas alternativos, evaluados para responder las siguientes preguntas de investigación: ¿Qué ocurre si aumenta la carga animal?, ¿Qué ocurre si se incrementa la suplementación? ¿Cómo afectan los sistemas de alimentación al resultado productivo y económico? ¿Qué ocurre si cambia el biotipo animal?, ¿Qué ocurre si se produce en un suelo BUENO o uno REGULAR?, ¿Qué ocurre si se produce en un área con mayor o menor precipitación?.

\section{Modelos de simulación utilizados}

En el presente estudio, el enfoque elegido para el análisis fue la simulación con el uso de modelos matemáticos. Se utilizaron dos modelos matemáticos: uno para predicción del rendimiento de los cultivos denominado APSIM (McCown, 1996; Keating et al., 2003), y otro para evaluación del sistema lechero completo, denominado e-Dairy (Baudracco et al., 2013). Los modelos se utilizaron para realizar simulaciones determinísticas y simulaciones estocásticas. Las simulaciones determinísticas son aquellas en las que todos los datos de entrada se mantienen fijos, produciendo un único resultado, mientras que las simulaciones estocásticas permiten incorporar variación en algunos parámetros, y así producir resultados múltiples, lo que permite el análisis del riesgo. En las simulaciones estocásticas se incorporó variación para el precio de la leche y de los concentrados y para el rendimiento de los forrajes, i.e., como respuesta a la variabilidad climática.

\section{Simulación de rendimiento y variabilidad de los cultivos con el modelo APSIM}

Los sistemas lecheros de la región bajo análisis utilizan principalmente pasturas de alfalfa en pastoreo y cultivos de maíz y sorgo para confección de ensilaje. La productividad de las pasturas de alfalfa y de los cultivos presentan variaciones importantes entre años, debido principalmente a variaciones en las precipitaciones.

El modelo APSIM ha sido calibrado en diversas regiones del mundo para predecir el rendimiento potencial y variación interanual de los cultivos anuales y perennes, incluyendo trabajos de calibración realizados en Argentina (Jáuregui et al., 2015). En el presente estudio, este modelo se utilizó para predecir el rendimiento de cultivos utilizando datos de suelos de la región y una serie histórica de temperatura máxima, temperatura mínima, radiación solar y precipitación de la región. Los resultados permitieron obtener coeficientes de variabilidad interanual de rendimientos de cultivos. Los resultados de las simulaciones con el modelo APSIM se utilizaron como inputs del modelo de simulación e-Dairy, para generar simulaciones estocásticas (aleatorias) en las que el rendimiento de los cultivos varíe aleatoriamente entre simulaciones.

\section{Modelo de simulación de sistema lechero (e-Dairy)}

Se utilizó un modelo de simulación de sistemas lecheros, dinámico y estocástico denominado e-Dairy (Baudracco et al., 2013). El modelo e-Dairy simula diariamente la producción de leche, grasa y proteína, peso vivo y condición corporal durante un periodo de 365 días para vacas individuales, y el resultado económico del sistema lechero. El rodeo se genera aleatoriamente a través de operaciones matriciales utilizando varianzas y covarianzas de las variables productivas. El modelo puede simular, estocásticamente, el comportamiento de variables clave del sistema lechero, tales como la cantidad de pastura producida y ofrecida, el mérito genético y los precios de la leche y de los suplementos exógenos. Los principales datos requeridos para correr el modelo son: uso del suelo, tipo de pastura, tasa de crecimiento mensual de pastura, cantidad y distribución de suplementos, calidad de los alimentos (EM y FDN), descripción del rodeo (número de vacas, 
patrón de pariciones), estructura de edad, condición corporal inicial (escala 1-5), peso vivo, probabilidades de preñez por servicio, merito genético (potencial de producción de leche, grasa y proteína) y datos económicos relacionados a los ingresos, egresos y al capital invertido. Los principales datos de salida del modelo e-Dairy son: consumo de pastura, reservas y concentrados por vaca, utilización anual de la pastura, producción de leche, cambios en la condición corporal de las vacas, resultado económico y rentabilidad. El modelo e-Dairy fue inicialmente diseñado para sistema pastoriles. Posteriormente se incorporó un módulo para predecir sistemas en confinamiento, como el sistema 6 (CORRAL) que se muestra en la Tabla 1.

Para simular numerosas situaciones probables, se realizaron 500 corridas aleatorias para cada uno de los 10 sistemas. Esto significa que se simularon 500 posibles años con diferentes combinaciones de clima y de precio de leche y de concentrados.

\section{Supuestos utilizados en la simulación}

Se asumieron supuestos productivos y económicos para los 10 sistemas productivos, los cuales se detallan a continuación.

\section{Superficie y animales}

Las simulaciones se realizaron para representar un sistema de 150 ha destinadas a vacas totales (vacas ordeño + vacas secas). Se asumió que la recría de hembras se realizó fuera de las 150 ha. La cantidad de VT utilizadas para simular cada sistemas surgen de la multiplicación de las 150 ha de superficie por la carga animal de cada sistema. Por ejemplo, en el sistema 2, con 1,3 VT ha-1 ${ }^{-1}$ la cantidad de VT fue de 195 (Tabla 1). La concentración de grasa butirosa en leche fue de $3,66 \%$ y la de proteína bruta de 3,29\%, según datos provistos por una industria de la región. La única excepción fue el sistema 5, con biotipo animal de genética denominada GENIAL (ver Tabla 1), para el cual se utilizó una concentración de grasa butirosa de $3,48 \%$ y de proteína bruta de $3,42 \%$. Dicho biotipo es producto de un programa de mejoramiento genético realizado en Argentina, con un enfoque similar al utilizado en Nueva Zelanda (Lopez Villalobos y Garrik, 2005).

De las 150 ha totales, en sistemas con suelo BUENO se destinó el 60\% para pastura de alfalfa, $17,5 \%$ para el doble cultivo avena/maíz para ensilaje, $17,5 \%$ sorgo para ensilaje, y $5 \%$ de superficie improductiva (instalaciones, callejones, etc.). En sistemas con suelo REGULAR se utilizó $67,5 \%$ para pastura de alfalfa, $13,75 \%$ para avena y luego maíz para ensilaje, $13,75 \%$ sorgo para ensilaje, y 5\% de superficie improductiva.

En los sistemas pastoriles se consideraron pérdidas anuales de vacas de $25 \%$ debido a mortandad y descartes, y de $29 \%$ para el sistema con alimentación a corral, basado en datos reportados por Candioti et al. (2013) y en el aporte de experiencia del equipo técnico. La mortandad de terneros asumida para periparto y los primeros dos meses de crianza fue de $10 \%$, igual para todos los sistemas.

\section{Rendimiento, calidad y eficiencia de uso de los forrajes}

El rendimiento de los cultivos y pasturas, en $\mathrm{kg}$ de MS por ha por año, se detalla en la Tabla 1. La calidad de los alimentos, definida por la concentración energética, fue de 2,27 megacalorías (Mcal) de energía metabólica $\mathrm{kg}$ $\mathrm{MS}^{-1}$ para la pastura de alfalfa, 2,17 Mcal kg MS ${ }^{-1}$ para el promedio de ensilajes de maíz y sorgo y de 2,82 Mcal kg MS-1 para el concentrado. Estos datos surgen de promedios de análisis de alimentos realizados en la región bajo estudio.

En relación a la eficiencia de uso de los alimentos, se asumió que las reservas y los concentrados ofrecidos en forma mezclada en comederos (en todos los sistemas excepto en el sistema 4) se consumieron con un $85 \%$ de eficiencia (consumido $\div$ ofrecido $\times 100$ ), y los concentrados ofrecidos en sala de ordeño se consumieron con una eficiencia del 95\% (sistema 4). La eficiencia de uso de ensilaje en autoconsumo se asumió en $85 \%$. La eficiencia de uso de las pasturas, también denominada eficiencia de cosecha (consumido $\div$ ofrecido $\times 100$ ), es un dato de salida del modelo e-Dairy, y fue estimada en cada situación por dicho modelo.

\section{Ingresos por venta de leche}

El precio de la leche utilizado y la desviación estándar (DE) para las simulaciones fue de \$US 0,35 litro $^{-1}(\mathrm{DE}=0,07)$ excepto en el sistema 5 , con vacas biotipo GENIAL, con un precio de \$US 0,36 litro $^{-1}(\mathrm{DE}=0,08)$, calculado en base al sistema de pago de la industria.

La simulación estocástica, con un promedio de \$US 0,35 litro $^{-1}(\mathrm{DE}=0,07)$, utilizando una distribución normal, genera un rango de precios entre \$US 0,10 y 0,60 por litro de leche.

\section{Ingresos por venta de carne}

Por la venta de las vacas de descarte se tomó un precio de $\$ U S 1,2 \mathrm{~kg}^{-1}(\mathrm{DS}=0,22)$ en pie. Por venta de terneros machos de una semana de vida se tomó un precio de $\$$ US 20 (DS =3,27) cabeza ${ }^{-1}$. Dichos valores corresponden a los precios promedio pagados en los mercados de carne de la región bajo estudio. 


\section{Gastos en alimentación}

El precio de los concentrados fue de \$US 0,22 $\mathrm{kg}^{-1}(\mathrm{DS}=0,05)$, con la variación calculada en base a una serie histórica de precios (AACREA, 2016). La excepción fue el precio de concentrados para el sistema confinado (CORRAL), con un precio de \$US $0,24 \mathrm{~kg}^{-1}$, debido a un mayor porcentaje de proteína, a causa de la mayor producción de leche por vaca de dicho sistema.

Para la implantación de pasturas se asumió un gasto de \$US 330 ha $^{-1}$, amortizado en 3 años, para mantenimiento de pasturas (herbicidas, fertilizantes y desmalezado) \$US $231 \mathrm{ha}^{-1}$ por año. El gasto para realizar cultivos para ensilaje fue de \$US 343 ha $^{-1}$ (promedio de maíz y sorgo) y para cultivos de invierno el gasto fue de \$US 198 ha $^{-1}$. Para el ensilaje se asumió un gasto de confección de \$US $0,058 \mathrm{~kg}^{-1}$ MS. Para la recría de hembras fuera del sistema, desde los dos meses de edad hasta dos meses previos al parto, se asumió un costo de pastaje de \$US 38 por cabeza por mes.

\section{Gastos de suministro de alimentos}

Se asumió un gasto de $\$$ US $0,051 \mathrm{~kg}^{-1} \mathrm{MS}$. Esto incluye el costo de amortización del tractor y del mixer, costos de reparación y mantenimiento, de combustible y de mano de obra. Se asumió que todos los sistemas suministran el concentrado y el ensilaje con mixer, excepto el sistema 4, el cual suministra el alimento concentrado en la sala de ordeño y el ensilaje en autoconsumo.

\section{Gastos relacionados a los animales y su manejo}

Para retribución a la mano de obra se consideró un $13 \%$ del ingreso por venta de leche en todos los sistemas. Los gastos de sanidad por vaca fueron de \$US $70 \mathrm{VT}^{-1}$ por año, de inseminación y control lechero de \$US $33 \mathrm{VT}^{-1}$ por año, de limpieza y mantenimiento de ordeñadora de \$US $36 \mathrm{VT}^{-1}$ por año y de electricidad de \$US 0,476 por litro de leche. Por la crianza de terneros se consideró un gasto de \$US 55 por ternero por mes de crianza, hasta los 60 días de edad. Se consideraron \$US 12 por vaca por año de gastos de tratamiento y aplicación de efluentes por vaca por año. Finalmente se consideraron \$US $13 \mathrm{VT}^{-1}$ por año por gastos directos varios.

\section{Alquiler de la tierra}

Se consideró que se pagó alquiler por la totalidad de la tierra de la lechería (150 ha), en todos los sistemas, con un monto equivalente a 80 litros de leche por ha por mes en los sistemas con suelo BUENO y 60 litros de leche por ha por mes en los sistemas con suelo REGULAR, de acuerdo a la información provista por productores $\mathrm{y}$ asesores del equipo técnico. Para los sistemas en los que se compró heno, se asumió un gasto de \$US 0,16 $\mathrm{kg}^{-1}$ MS comprada.

\section{Gastos indirectos}

Los siguientes gastos indirectos son comunes a todos los sistemas simulados. Por reparaciones y mantenimiento de maquinarias y mejoras \$US 47 ha $^{-1}$ por año, administración y asesoramiento \$US 58 ha $^{-1}$ por año. Por amortizaciones totales se computó \$US $155 \mathrm{ha}^{-1}$ por año, que se calculó en base al valor a nuevo y la duración de maquinarias y mejoras. Por impuestos, tasas y seguros se computaron \$US $105 \mathrm{ha}^{-1}$ por año. Por gastos varios indirectos se computó \$US $30 \mathrm{ha}^{-1}$ por año.

\section{Inversiones}

Se asumió una inversión de \$US 1.126 por vaca total en todos los sistemas, lo que corresponde a instalaciones de ordeño, equipo de ordeño y equipos de refrigeración de la leche. La inversión por maquinarias (extra suministro de alimento) y mejoras fue de \$US 804, excepto en el sistema CORRAL (6), con animales confinados, en el que fue menor (\$US 751) por tener menos callejones y tener las aguadas concentradas en una superficie reducida. La inversión necesaria para confinamiento de animales en el sistema 6 fue de \$US 900 (levante de superficie con tierra, instalación de sombras y pista de cemento para sector de alimentación).

Para todos los sistemas pastoriles (todos excepto el sistema 6) se asumió una inversión extra de \$US 70 por vaca para una pista de alimentación para ser usada en periodos de excesos hídricos. Para el sistema 4 se asumió una inversión extra de \$US 110 por vaca por la instalación de racionadores automáticos para suministro de concentrado en la sala de ordeño.

\section{Simulaciones determinísticas y estocásticas de los 10 sistemas productivos}

Se realizó la simulación de los 10 sistemas a escala de lechería comercial mediante el uso del software e-Dairy. Se evaluaron tres escenarios, i) determinístico, en el que se obtiene un resultado único para cada sistema (precio de leche y concentrados y rendimientos de cultivos fijos), y dos escenarios de incertidumbre o riesgo, ii) un escenario con variación aleatoria del precio de la leche y el precio del alimento concentrado, $y$ iii) un escenario de precios fijos, pero de rendimientos variables de todos los cultivos.

En el escenario de precios variables, el objetivo fue investigar el efecto que tiene el mercado sobre el riesgo de cada uno de los 10 sistemas simulados. En el escenario de rendimientos de cultivos variables, el objetivo fue investigar el 
efecto del clima sobre la producción de leche y el resultado económico a través del efecto del clima sobre los rendimientos de los cultivos (alfalfa, avena, maíz y sorgo).

\section{RESULTADOS Y DISCUSIÓN}

\section{Resultados productivos y económicos: \\ Simulación determinística.}

Elpresenteestudiofuediseñadoparainvestigar alternativas de sistemas productivos en la región Centro-Norte de Argentina. Los resultados de las simulaciones determinísticas, i.e., precios de la leche y de los concentrados y rendimientos de cultivos y pasturas fijos, se muestran en la Tabla 2. Se observa que el sistema BASE (sistema 1), diseñado para representar la situación actual de la región bajo estudio, obtuvo una producción de leche de 6.026 litros ha-1 por año, lo cual es inferior al promedio de Argentina, de 7.580 litros ha $^{-1}$ por año (Ministerio de Agroindustria de la Nación, 2016). Todas las alternativas evaluadas en suelos BUENOS produjeron más leche por ha que el sistema BASE. Los únicos sistemas que produjeron menos que el sistema BASE son los sistemas sobre suelo REGULAR y con baja oferta de suplementos externos (sistema 7 y 8 ).

El resultado económico del sistema BASE

Tabla 2. Descripción de los sistemas modelados y de los principales resultados productivos y económicos de los 10 sistemas lecheros simulados.

Table 2. Description and main productive and economic results of the 10 modeled dairy systems.

\begin{tabular}{|c|c|c|c|c|c|c|c|c|c|c|}
\hline & 1 & 2 & 3 & 4 & 5 & 6 & 7 & 8 & 9 & 10 \\
\hline \multicolumn{11}{|l|}{ Descripción de sistemas } \\
\hline Precipitación & Baja & Baja & Baja & Baja & Baja & Baja & Baja & Alta & Alta & Alta \\
\hline Suelo & Bueno & Bueno & Bueno & Bueno & Bueno & Bueno & Regular & Regular I & Regular & Bueno \\
\hline Sistema alimentación & Pasto & Pasto & Pasto & Autoc. & Pasto & Corral & Pasto & Pasto & Pasto & Pasto \\
\hline Tipo vaca & H580 & H580 & H580 & H580 & Genial & H700 & $\mathrm{H} 580$ & H580 & H580 & H580 \\
\hline Carga animal & 1,0 & 1,3 & 1,6 & 1,6 & 1,7 & 1,7 & 0,8 & 0,8 & 1,5 & 1,3 \\
\hline $\begin{array}{l}\text { Concentrado } \\
\text { (kg MS vaca }{ }^{-1} \text { por día) }\end{array}$ & 4,0 & 5,5 & 7,0 & 7,0 & 7,0 & 10,0 & 4,0 & 4,0 & 7,0 & 5,0 \\
\hline \multicolumn{11}{|l|}{ Resultados productivos } \\
\hline Eficiencia uso pastura & $62 \%$ & $66 \%$ & $67 \%$ & $66 \%$ & $65 \%$ & - & $66 \%$ & $62 \%$ & $67 \%$ & $54 \%$ \\
\hline $\begin{array}{l}\text { Pastura consumida } \\
\text { (kg MS VT-1 por año) }\end{array}$ & 2653 & 2212 & 1874 & 1842 & 1727 & 0 & 2271 & 2299 & 1363 & 2450 \\
\hline $\begin{array}{l}\text { Reservas consumidas } \\
\text { (kg MS VT-1 por año) }\end{array}$ & 1988 & 1984 & 1993 & 1998 & 1986 & 3726 & 2371 & 2483 & 2825 & 2190 \\
\hline $\begin{array}{l}\text { Concentrado consumido } \\
\text { (kg MS VT }{ }^{-1} \text { por año) }\end{array}$ & 1308 & 1710 & 2112 & 2228 & 2112 & 2912 & 1313 & 1307 & 2143 & 1576 \\
\hline Litros leche $\mathrm{VO}^{-1}$ por día & 20,1 & 20,2 & 21,2 & 21,8 & 21,2 & 26,1 & 18,4 & 19,1 & 21,7 & 21,9 \\
\hline $\begin{array}{l}\text { Litros leche ha-1 por año } \\
\mathrm{kg} \text { Sólidos ha- }{ }^{-1} \text { por año }\end{array}$ & 6026 & 7911 & 10235 & 10507 & 10856 & 13250 & 4421 & 4584 & 9709 & 8576 \\
\hline$(\mathrm{kg} \mathrm{GB}+\mathrm{P} \mathrm{B})$ & 419 & 553 & 717 & 736 & 750 & 925 & 306 & 318 & 680 & 599 \\
\hline \multicolumn{11}{|l|}{$\begin{array}{l}\text { Resultados económicos } \\
\text { (\$US ha-1 por año) }\end{array}$} \\
\hline Ingreso total & 2267 & 2974 & 3705 & 3800 & 3897 & 4950 & 1764 & 1873 & 3340 & 3208 \\
\hline Gastos directos & 1568 & 1990 & 2478 & 2364 & 2594 & 3558 & 1368 & 1396 & 2360 & 2025 \\
\hline Gastos indirectos & 684 & 684 & 684 & 684 & 684 & 684 & 600 & 600 & 600 & 684 \\
\hline Resultado económico & 15 & 300 & 543 & 752 & 619 & 708 & -204 & -123 & 380 & 499 \\
\hline Capital invertido & 539034 & 693594 & 849833 & 870990 & 900796 & 1190101 & 435163 & 438025 & 798811 & 700277 \\
\hline Rentabilidad, \% & 0,4 & 6,5 & 9,6 & 12,9 & 10,3 & 8,9 & $-7,0$ & $-4,2$ & 7,1 & 10 \\
\hline
\end{tabular}

Resultado económico $\left(\$ \mathrm{US} \mathrm{ha}^{-1}\right.$ por año) $=$ Ingresos leche + ingreso carne $-($ gastos directos + gastos indirectos + amortizaciones + alquiler de tierra).

Capital invertido: es todo el capital invertido, incluyendo animales y excluyendo tierra, en las 150 ha.

Rentabilidad: Resultado económico dividido el capital invertido. 
es prácticamente neutro con \$US $15 \mathrm{ha}^{-1}$ por año (Tabla 2). Todos las alternativas evaluadas presentaron mayor resultado económico que el sistema BASE, excepto los dos sistemas simulados en suelo REGULAR, con baja producción de forrajes y baja suplementación (sistemas 7 y 8). El sistema 9, en suelo REGULAR y con mayor suplementación, también tuvo mejores resultados que el sistema BASE, Esto muestra que existe posibilidad de aumentar la productividad y mejorar el resultado económico de los sistemas actuales de la zona de estudio.

Las producciones de leche por vaca, son el resultado de la predicción del modelo e-Dairy, en función de la oferta y calidad de alimentos y de la demanda animal. En todos los sistemas, y particularmente en el sistema 6 (CORRAL), se podrían obtener producciones de leche por vaca más altas que las obtenidas en el presente estudio, con mayor oferta y más calidad de alimentos. En el presente estudio, la oferta de alimentos por fue relativamente baja, debido a que se investigó una región con limitaciones para producción de forrajes.

\section{Carga animal}

La carga animal es la práctica de manejo con mayor influencia en la eficiencia de los sistemas de base pastoril (Holmes y Roche, 2007). En este estudio, todos los sistemas con carga animal más alta que el sistema $\operatorname{BASE}(3,4,5$ y 6), en suelos BUENOS, tuvieron mayor producción de leche por hectárea y mayores resultados económicos y rentabilidad que los sistemas de carga animal más baja en los mismos suelos.

Esto coincide con estudios previos en Argentina (Baudracco et al., 2011) y en otros países con sistemas lecheros pastoriles (Macdonald et al., 2008; 2011), en los que se reportó que el aumento de la carga animal incrementó la productividad de leche por ha y el resultado económico. En el caso del estudio de Macdonald, se evaluaron cargas animales de 2,2 a 4,2 VT ha-1 en Nueva Zelanda, y el aumento de la carga animal generó siempre mayores producciones de leche por ha, pero el máximo resultado económico se observó a una carga animal intermedia. En dicho estudio no se utilizó suplementación, y los niveles más altos de carga animal evaluados fueron tan altos que afectaron la producción de leche por vaca, incrementando más los gastos por vaca que el ingreso por leche extra.

En contraste, en el estudio que evaluó el efecto de la carga animal acompañada de mayor suplementación por ha en Argentina (Baudracco et al., 2011), se reportó que el incremento en la carga animal (de 1,6 a 2,6 VT ha-1) mejoró la utilización de las pasturas, generó mayor producción de leche por hectárea y mejoró el resultado económico, debido a que la mayor producción de leche por ha generó más ingresos que gastos. Similarmente, en el presente estudio, las cargas más altas que el sistema BASE (sistemas 3, 4, 5 y 6) generaron mejores resultados económicos. Tanto en el estudio de Baudracco et al. (2011) como en el presente estudio, el incremento de la carga animal estuvo acompañado de mayor suplementación por vaca, lo que permitió que la producción de leche por vaca no disminuya con el aumento de la carga animal. En la región bajo estudio, sería recomendable entonces incrementar la carga animal (actualmente baja: 1,0 VT ha ${ }^{-1}$ ) y la suplementación, manteniendo la producción individual de leche por vaca, para lograr incrementos en el resultado económico.

\section{Suplementación}

Los resultados muestran que fue conveniente incrementar la suplementación con concentrados por vaca, respecto al sistema BASE, siempre y cuando se mantenga la eficiencia de cosecha de pastura por un incremento simultáneo de la carga animal. La inclusión de suplementos en los sistemas lecheros afecta el consumo de pastura, y consecuentemente, la productividad y rentabilidad del sistema. Bajas cargas animales permiten altas producciones de leche por vaca, pero conducen a un bajo aprovechamiento de pasturas, mientras que altas cargas animales conducen a bajas producciones de leche por vaca (Holmes y Roche, 2007). Los suplementos suministrados en sistemas con necesidad de alimento, como aquellos con carga animal alta, pueden potencialmente permitir que se logre el doble objetivo de mantener buena performance individual (vacas productivas y sanas) y a su vez permitir que las pasturas sean utilizadas en forma eficiente (Macdonald et al., 2008).

El uso de suplementos puede incrementar la eficiencia de utilización de la pastura en el sistema, dado que permite incrementar la presión sobre las pasturas a partir de un aumento de la carga animal, como se observa en los sistemas 3, 4, 5 y 6, comparados con el sistema BASE de menor carga animal. El incremento simultáneo de la carga animal y de la suplementación pueden mejorar la performance del sistema lechero (Holmes y Roche, 2007), como pudo determinarse en el presente estudio (Tabla 2).

\section{Sistemas de alimentación}

La mayor producción de leche por vaca se logró en el sistema 6 con 26,1 litros de leche $\mathrm{VO}^{-1}$ por día, debido a que fue el sistema que más alimento ofreció por vaca. Este sistema tuvo además la carga animal más alta $\left(1,7 \mathrm{VT} \mathrm{ha}^{-1}\right)$, y produjo 
la mayor cantidad de leche por ha, con 13.250 litros ha ${ }^{-1}$ por año, el doble de productividad que el sistema BASE. Este sistema (6), a pesar de ser el de mayor producción de leche por ha y uno de los de mayor resultado económico, se ubicó en quinto lugar en rentabilidad (resultado económico $\div$ capital invertido), debido a que fue el que requirió la mayor cantidad de capital invertido (Tabla 2).

El sistema 4, idéntico al sistema 3 pero con alimentación de ensilaje en autoconsumo y alimento concentrado en la sala de ordeño, tuvo un desempeño productivo y económico levemente superior al sistema 3, debido a que tuvo mayor eficiencia de uso de concentrado y menores costos de suministro de alimentos.

\section{Biotipo animal}

El sistema 5, con el biotipo animal GENIAL, fue idéntico al sistema 3, excepto por el biotipo animal. El sistema 5 tuvo un desempeñolevemente superior al sistema 3 debido a que, al ser la vaca tipo GENIAL más liviana, se pudo incrementar levemente la carga animal y así aprovechar los beneficios de este incremento en la carga, como se mencionó anteriormente. Además, se asumió un precio de leche levemente superior para este biótico, debido al mayor contenido proteico de la leche.

\section{Tipo de suelo y precipitación}

Los resultados indican que en los sistemas con suelo REGULAR y con baja suplementación (sistemas 7 y 8, Tabla 2), que producen menor cantidad de forraje, la producción de leche por ha y los resultados económicos son inferiores a los obtenidos en sistemas con suelos BUENOS. Es decir que, aunque en estos sistemas el gasto en alquiler de la tierra sea inferior porque la tierra es de inferior calidad, esa reducción de gasto no se traduce en un beneficio económico, porque se produce poco forraje y poca leche. Una alternativa rentable en dichos suelos sería incrementar la compra de alimentos para mejorar la oferta de forrajes y la producción de leche. Esto lo muestra el sistema 9, que tiene resultados económicos positivos aun en un suelo REGULAR, debido a que incrementó la producción de leche comparado con los sistemas 7 y 8 , debido a la compra de reservas (heno) y mayor cantidad de concentrados.

Los resultados de la simulación indican que en suelos de calidad REGULAR no se observaron ventajas por un incremento en la precipitación. Esto se deduce al comparar dos sistemas idénticos (excepto por la precipitación): el sistema 7 de suelo REGULAR y precipitación BAJA con el sistema 8 de suelo REGULAR y precipitación ALTA, ambos con resultado económico negativo (Tabla 2). Sin embargo, el efecto de la precipitación es más notorio en sistemas con suelos BUENOS. Esto se observa al comparar el sistema 2 con el sistema 10, ambos idénticos excepto por la precipitación. Allí se observa que el sistema 10, con precipitación ALTA supera en resultado económico al sistema 2, con precipitación BAJA.

\section{Riesgo de mercado y de clima: Simulación estocástica}

Como se detalló en la metodología, se realizaron para cada sistema 500 simulaciones, para evaluar el efecto de mercado. En cada simulación de un sistema determinado, se aleatorizó el precio de la leche y del concentrado. Igualmente, se realizaron 500 simulaciones por sistema para evaluar el efecto del clima, aleatorizando en cada corrida los rendimientos de los cultivos. En la Fig. 1 se muestra el rango de resultados posibles para cada sistema en los escenarios de incertidumbre de clima (Fig. 1A) y de mercado (Fig. 1B).

Para todos los sistemas evaluados, el efecto de mercado (variación de precio de leche y concentrado) fue mayor que el efecto del clima (variación del rendimiento de los cultivos) sobre el resultado económico de los sistemas (Fig. 1A y 1B).

El efecto de mercado fue más grande que el del clima, debido a que i) el efecto de mercado impacta directamente sobre el resultado económico, a diferencia del efecto del clima que impacta sobre la producción de los cultivos y luego indirectamente sobre la producción de leche, ii) el efecto de mercado actúa sobre dos factores clave, el precio de la leche y el precio del alimento concentrado, el cual representa el mayor gasto de los sistemas lecheros de Argentina. Así, en casos en que la aleatorización de precios combina bajo precio de leche y alto precio de concentrado el efecto es muy fuerte y viceversa, generando amplia variación de resultados (Fig. 1B).

El efecto del clima sobre los rendimientos de los cultivo impacta moderadamente sobre la producción de leche en sistemas pastoriles, porque en años climáticamente adversos hay menor producción de pastura y por ello la eficiencia de cosecha es mayor que en años con alta producción de pastura (climáticamente favorables), lo que atempera el efecto sobre la reducción en producción de leche (ver eficiencia de uso de pastura, en Tabla 2). A su vez, en años climáticamente favorables, hay mayor producción de pastura, la cual no puede ser aprovechada con eficiencia, debido a que la carga animal se mantiene constante dentro de un mismo sistema.

El sistema con menor variación (menor 

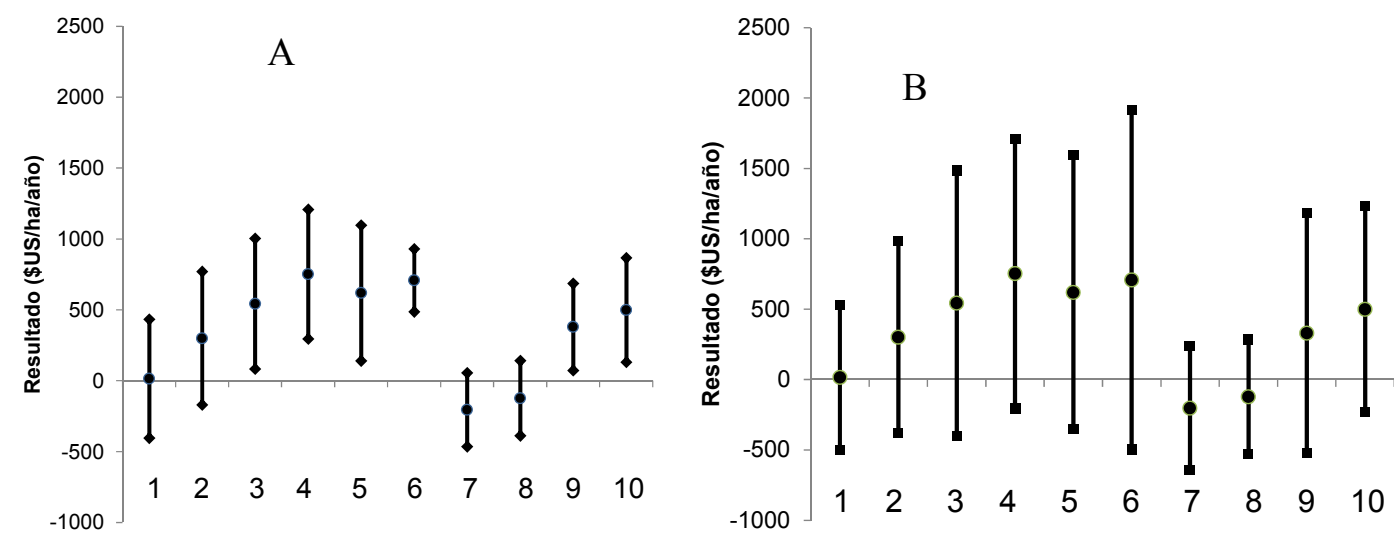

Fig. 1. Resultado económico esperado en cada uno de los 10 sistemas productivos investigados, frente a variaciones de rendimiento de cultivos debido al clima (A) y frente a variaciones en el precio de la leche y el precio de los concentrados (B). El círculo del medio de la barra indica el promedio y el largo de la barra indica el rango en el que se observarían los resultados.

Fig. 1. Expected economic results for each of the 10 dairy systems evaluated, exposed to variations in: crop and pasture yield (A) and concentrate feed prices (B). The circle in the center of the bar represents the average, and the length of the bar represents the range in which results can be expected.

riesgo) frente al efecto del clima (Fig. 1A) fue el sistema 6. Esto es debido a que tiene las vacas en confinamiento a corral y la base de la alimentación son ensilajes y concentrado. Este ha sido uno de los argumentos de productores de Argentina para confinar las vacas durante todo el año, suprimiendo el pastoreo y evitando la dependencia de los rendimientos de pastura, logrando mayor independencia del clima. Sin embargo, se muestra en la Fig. 1B, que este sistema es muy afectado por el efecto del mercado, es decir que es más estable al efecto climático pero más dependiente de los precios de la leche y los concentrados, debido a que es el sistema con mayor dependencia de los concentrados.

Los sistemas con mayor carga animal y mayor cantidad de concentrados (sistemas 3, 4, 5 y 6), presentaron mayor exposición frente al riesgo de mercado (Fig. 1B). La intensificación, a partir del incremento de la carga animal y de la suplementación, mejoró los resultados económicos pero aumentó la exposición al riesgo.

Los sistemas 1, 7 y 8 , sobre tierra alquilada y mano de obra contratada, como se consideró en la simulación, serían inviables, debido a que más de la mitad de los años obtendrían resultado económico negativo (Fig. 1).

\section{CONCLUSIONES}

Los resultados obtenidos son válidos bajo los supuestos declarados en la metodología del presente estudio, supuestos diferentes producirían resultados diferentes. El sistema BASE, que representa el sistema actual de la región, con 1,0 VT ha-1 y 6.026 litros de leche producidos por ha, sería inviable sobre tierra alquilada y con mano de obra contratada, como se consideró en la simulación, debido a que su resultado económico es cercano a cero.

El incremento de la carga animal, sobre el sistema BASE, manteniendo la producción de leche por vaca con más alimento concentrado por vaca, permitiría mejorar el resultado económico de los sistemas lecheros de la región.

En suelos de baja producción de forraje, no convendría producir leche, a menos que se importe al sistema una elevada cantidad de suplementos para incrementar la carga y la producción de leche por hectárea.

El sistema con alimentación a corral, según los supuestos utilizados en la simulación, produciría buenos resultados económicos, pero con alta exposición al efecto del mercado. El sistema de alimentación en autoconsumo y la genética animal GENIAL serían tecnologías que podrían mejorar los resultados económicos del sistema BASE de la región.

El efecto de mercado (variación de precio de leche y concentrado) es mayor que el efecto del clima (en el rendimiento de los cultivos), sobre el resultado económico de todos los sistemas evaluados. 


\section{AGRADECIMIENTOS}

A todas las instituciones que conforman el CLUSTER Lechero Regional (Asociación para el Desarrollo Regional de la Cadena Láctea del N. O. de Santa Fe y el S.E. de Santiago del Estero). A los técnicos de INTA San Cristóbal y de INTA Ceres y a la Cooperativa Lechera Nueva Alpina.

\section{LITERATURA CITADA}

AACREA, 2016. Series de precios agropecuarios. Disponible en http://www.aacrea.org.ar (Consulta: 12 Julio 2016)

Baudracco, J., N. Lopez-Villalobos, L.A. Romero, D. Scandolo, M.G. Maciel, E.A. Comeron, et al. 2011. Effects of stocking rate on pasture production, milk production and reproduction of supplemented crossbred Holstein-Jersey dairy cows grazing lucerne pasture. Animal Feed Science and Technology 168:131-143.

Baudracco, J., N. Lopez-Villalobos, C.W. Holmes, E.A. Comeron, K.A. Macdonald, and T.N. Barry. 2013. e-Dairy: a dynamic and stochastic whole-farm model that predicts biophysical and economic performance of grazing dairy systems. Animal 7:870-878.

Baudracco, J., B. Lazzarini, D. Lovino, E. Demarchi, y R. Giorgis. 2016. Bases para una producción de leche simple y rentable en Argentina. En Actas de XLIV Jornadas Uruguayas de Buiatría. 9-10 junio 2016. Sociedad de Medicina Veterinaria del Uruguay, Paysandu, Uruguay.

Beukes, P.C., C.C Palliser, K.A. Macdonald, J.A.S. Lancaster, G. Levy, B.S. Thorrold, et al. 2008. Evaluation of a whole-farm model for pasture-based dairy systems. Journal of Dairy Science 91:2353-2360.

Candioti, F., J. Baudracco, N. Bocco, L. Chapado, D. Manelli, F. Maranzana, et al. 2013. Comparación productiva y económica entre sistemas lecheros pastoriles y confinados de Argentina. En 3er Simposio Internacional Leite Integral. 8 y 9 de mayo 2013. Belo Horizonte, Brasil.

Gagliardi, R., y N. Lopez-Villalobbos. 2012. Genética bovina lechera en la Cooperativa Tambera Nueva Alpina Ltda. Revista Producir 20(248): 22-28

Holmes, C.W., and J.F. Roche. 2007. Pasture and supplements in New Zealand Dairy Production Systems. In Pastures and Supplements for grazing animals Occ. Pub. No 14. New Zealand Society of Animal Production, Hamilton, New Zealand.
Jáuregui, J.M., J.J. Ojeda, J. Baudracco, J. Maiztegui, C. Dimundo, J. Ibarlucea, et al. 2015. Calibración del modelo APSIM para evaluar el potencial productivo de alfalfa en el Noroeste de Santa Fe. Revista Argentina de Producción Animal 35:126.

Keating, B.A., P.S. Carberry, G.L Hammer, M.E Probert, M.J Robertson, D. Holzworth, et al. 2003. An overview of APSIM, a model designed for farming systems simulation. European Journal of Agronomy 18:267-288.

Lopez-Villalobos, N., and D.J. Garrick. 2005. Methodology for the design and enhancement of genetic improvement programs illustrated in the context of the New Zealand dairy industry. Agrociencia 9:553-568.

Macdonald K.A., J.W. Penno, J.A.S. Lancaster, and J.R. Roche. 2008. Effect of stocking rate on pasture production, milk production, and reproduction of dairy cows in pasture-based systems. Journal of Dairy Science 91:21512163.

Macdonald, K.A., D. Beca, J.W. Penno, J.A.S. Lancaster, and J.R. Roche. 2011. Effect of stocking rate on the economics of pasture based dairy farms. Journal of Dairy Science 94:2581-2586.

McCown, R.L., G.L. Hammer, J.N.G. Hargreaves., D.P. Holzworth, and D.M. Freebairn. 1996. APSIM: a novel software system for model development, model testing, and simulation in agricultural systems research. Agricultural Systems 50:255-271.

Ministerio de Agroindustria de la Nación Argentina. 2016. Disponible en http://www. agroindustria.gob.ar (Consulta 7 abril 2016). 